\title{
Generating of fuzzy implications
}

\author{
Anna Król ${ }^{1}$ \\ ${ }^{1}$ Institute of Mathematics, University of Rzeszów, annakrol@univ.rzeszow.pl
}

\begin{abstract}
This paper deals with some dependencies between fuzzy connectives, on the pattern of laws in a classical propositional calculus, which allow to generate fuzzy implications. Fuzzy implications generated both by a triple: fuzzy conjunction, disjunction and negation, and by two fuzzy implications are considered. In the case of a fuzzy conjunction and disjunction only border conditions and monotonicity are assumed.
\end{abstract}

Keywords: QL-implication, fuzzy implication, fuzzy conjunction, fuzzy disjunction, fuzzy negation

\section{Introduction}

In this contribution two ways of generating of fuzzy implication from other fuzzy connectives are considered. The first method allows to create fuzzy implications from a fuzzy conjunction, disjunction and negation. Implications created in such a way have been considered in the literature in the case when the conjunction is a triangular norm and the disjunction is a triangular conorm e.g. by M. Baczyński and B. Jayaram [1], Y. Shi et al. [7] and are called QL-implications. Other generalization of QL-implications were examined e.g. by M. Mas et al. [6], where a triangular norm and conorm where replaced by conjunctive and disjunctive uninorm, respectively. In this article assumption about commutativity, associativity and neutral element of the connectives is omitted. The need od such weak connectives and their use in fuzzy inference system has been indicated e.g. by I. Batyrshin and O. Kaynak [2].

The second method is connected with the equation $I(x, y)=I(x, I(x, y))$ which was considered e.g by Y. Shi et al. [8]. This formula allows us to generate a fuzzy implication from other two ones. The idea was firstly presented by the author [4]. It was also considered by N.R. Vemuri and B. Jayaram [9].

In the following section the definitions and examples of fuzzy connectives used in the sequel are presented. Next, in Section 3, fuzzy implications generated from a fuzzy conjunction, disjunction and negation are considered. Finally, Section 4 presents the results concerning preservation of some properties of fuzzy implication generated by two fuzzy implications.

\section{Preliminaries}

In this section some algebraic properties of binary operations as well as definitions and examples of fuzzy connectives used in the sequel are presented. First, properties of binary operation connected to the notion of zero element is recalled.

Definition 1. We say that an operation $A$ : $[0,1]^{2} \rightarrow[0,1]$ has

- a left zero element $z$ if $A(z, x)=z, x \in[0,1]$,

- a right zero element $z$ if $A(x, z)=z, x \in[0,1]$,

- a zero element $z$ if $A(z, x)=A(x, z)=z, x \in$ $[0,1]$.

Definition 2. Let $z$ be a zero element of operation $A:[0,1]^{2} \rightarrow[0,1]$. We say that $A$ is without zero divisors if

$$
A(x, y)=z \Rightarrow x=z \vee y=z,
$$

where $x, y \in[0,1]$.

Next, the notion of a fuzzy negation is considered.

Definition 3 ([1], p. 14). A decreasing function $N:[0,1] \rightarrow[0,1]$ is called a fuzzy negation if

$$
N(0)=1, \quad N(1)=0 .
$$

Definition 4 ([1], p. 14). A fuzzy negation is called a strong negation if it an involution, i.e. it fulfils the condition $N(N(x))=x, x \in[0,1]$.

Example 1. Let us focus on the standard fuzzy negation $N_{S}(x)=1-x$ for $x \in[0,1]$, the least fuzzy negation $N_{0}$ and the greatest fuzzy negation $N_{1}$, where

$$
\begin{aligned}
& N_{0}(x)=\left\{\begin{array}{ll}
1, & \text { if } x=0 \\
0, & \text { if } x>0
\end{array},\right. \\
& N_{1}(x)=\left\{\begin{array}{ll}
1, & \text { if } x<1 \\
0, & \text { if } x=1
\end{array} .\right.
\end{aligned}
$$

Now, the definition of a fuzzy conjunction is presented.

Definition $5([3])$. An operation $C:[0,1]^{2} \rightarrow[0,1]$ is called a fuzzy conjunction if it is increasing with respect to each variable and

$$
C(1,1)=1, \quad C(0,0)=C(0,1)=C(1,0)=0 .
$$

Corollary 1. A fuzzy conjunction has a zero element 0 . 
Example 2. Let us consider the following family of fuzzy conjunctions for $\alpha \in[0,1]$

$$
C_{\alpha}(x, y)= \begin{cases}1, & \text { if } x=y=1 \\ 0, & \text { if } x=0 \text { or } y=0 . \\ \alpha & \text { otherwise }\end{cases}
$$

Operations $C_{0}$ and $C_{1}$ are the least and the greatest fuzzy conjunction, respectively, where

$$
\begin{gathered}
C_{0}(x, y)=\left\{\begin{array}{ll}
1, & \text { if } x=y=1 \\
0, & \text { otherwise }
\end{array},\right. \\
C_{1}(x, y)= \begin{cases}0, & \text { if } x=0 \text { or } y=0 \\
1, & \text { otherwise }\end{cases}
\end{gathered}
$$

The following are the other examples of fuzzy conjunctions. Triangular norms are denoted in the traditional way.

$$
\begin{aligned}
C_{2}(x, y) & =\left\{\begin{array}{ll}
y, & \text { if } x=1 \\
0, & \text { if } x<1
\end{array},\right. \\
C_{3}(x, y) & =\left\{\begin{array}{ll}
x, & \text { if } y=1 \\
0, & \text { if } y<1
\end{array},\right. \\
C_{4}(x, y) & =\left\{\begin{array}{ll}
0, & \text { if } x+y \leqslant 1 \\
y, & \text { if } x+y>1
\end{array},\right. \\
C_{5}(x, y) & =\left\{\begin{array}{ll}
0, & \text { if } x+y \leqslant 1 \\
x, & \text { if } x+y>1
\end{array},\right. \\
T_{M}(x, y) & =\min (x, y), \\
T_{P}(x, y) & =x y, \quad \text { max }(x+y-1,0), \\
T_{L K}(x, y) & =\left\{\begin{array}{ll}
x, & \text { if } y=1 \\
y, & \text { if } x=1 \\
0, & \text { otherwise }
\end{array},\right. \\
T_{D}(x, y) & \text { if } x+y \leqslant 1 \\
T_{n M}(x, y) & = \begin{cases}0, & \text { otherwise } \\
\min (x, y),\end{cases}
\end{aligned}
$$

Next, the notion of a fuzzy disjunction is recalled.

Definition $6([3])$. An operation $D:[0,1]^{2} \rightarrow[0,1]$ is called a fuzzy disjunction if it is increasing with respect to each variable and

$$
D(0,0)=0, \quad D(0,1)=D(1,0)=D(1,1)=1 .
$$

Corollary 2. A fuzzy disjunction has a zero element 1 .

Example 3. Consider the following family of fuzzy disjunctions for $\alpha \in[0,1]$

$$
D_{\alpha}(x, y)= \begin{cases}0, & \text { if } x=y=0 \\ 1, & \text { if } x=1 \text { or } y=1 \\ \alpha & \text { otherwise }\end{cases}
$$

Operations $D_{0}$ and $D_{1}$ are the least and the greatest fuzzy disjunction, respectively, where

$$
\begin{gathered}
D_{0}(x, y)=\left\{\begin{array}{ll}
1, & \text { if } x=1 \text { or } y=1 \\
0 & \text { otherwise }
\end{array},\right. \\
D_{1}(x, y)=\left\{\begin{array}{ll}
0, & \text { if } x=y=0 \\
1 & \text { otherwise }
\end{array} .\right.
\end{gathered}
$$

The following are other examples of fuzzy disjunctions. Triangular conorms are denoted in the traditional way.

$$
\begin{aligned}
D_{2}(x, y) & =\left\{\begin{array}{ll}
y, & \text { if } x=0 \\
1, & \text { if } x>0
\end{array},\right. \\
D_{3}(x, y) & =\left\{\begin{array}{ll}
x, & \text { if } y=0 \\
1, & \text { if } y>0
\end{array},\right. \\
D_{4}(x, y) & =\left\{\begin{array}{ll}
1, & \text { if } x+y \geqslant 1 \\
y, & \text { if } x+y<1
\end{array},\right. \\
D_{5}(x, y) & =\left\{\begin{array}{ll}
1, & \text { if } x+y \geqslant 1 \\
x, & \text { if } x+y<1
\end{array},\right. \\
S_{M}(x, y) & =\max (x, y), \\
S_{P}(x, y) & =x+y-x y, \\
S_{L K}(x, y) & =\min (x+y, 1), \\
S_{D}(x, y) & =\left\{\begin{array}{ll}
x, & \text { if } y=0 \\
y, & \text { if } x=0 \\
1 & \text { otherwise }
\end{array},\right. \\
S_{n M}(x, y) & =\left\{\begin{array}{ll}
1, & \text { if } x+y \geqslant 1 \\
\max (x, y), & \text { otherwise }
\end{array} .\right.
\end{aligned}
$$

Finally, the notion of a fuzzy implication is recalled.

Definition 7 ([1], p. 2). A binary operation $I:[0,1]^{2} \rightarrow[0,1]$ is called a fuzzy implication if it is decreasing with respect to the first variable and increasing with respect to the second variable and

$$
I(0,0)=I(0,1)=I(1,1)=1, \quad I(1,0)=0 .
$$

Corollary 3. A fuzzy implication has a right zero element 1 and fulfils the condition

$$
I(0, y)=1, \quad x, y \in[0,1]
$$

Definition 8 ([1], pp. 9, 20). We say that a fuzzy implication $I$ satisfies:

- the neutral property (NP) if

$$
I(1, y)=y, \quad y \in[0,1]
$$

- the exchange principle (EP) if

$$
I(x, I(y, z))=I(y, I(x, z)), \quad x, y, z \in[0,1],
$$


- the identity principle (IP)

$$
I(x, x)=1, \quad x \in[0,1],
$$

- the ordering property (OP) if

$$
I(x, y)=1 \Leftrightarrow x \leqslant y, \quad x, y \in[0,1]
$$

Example 4 ([1], pp. 4,5). Let us present the following family of fuzzy implications for $\alpha \in[0,1]$

$$
I^{\alpha}(x, y)= \begin{cases}0, & \text { if } x=1, y=0 \\ 1, & \text { if } x=0 \text { or } y=1 \\ \alpha & \text { otherwise }\end{cases}
$$

The operations $I_{0}$ and $I_{1}$ are the least and the greatest fuzzy implication, respectively, where

$$
\begin{aligned}
& I_{0}(x, y)=\left\{\begin{array}{ll}
1, & \text { if } x=0 \text { or } y=1 \\
0, & \text { otherwise }
\end{array},\right. \\
& I_{1}(x, y)= \begin{cases}0, & \text { if } x=1, y=0 \\
1, & \text { otherwise }\end{cases}
\end{aligned}
$$

The following are the other examples of fuzzy implications.

$$
\begin{aligned}
I_{\mathrm{EK}}(x, y) & =\min (1-x+y, 1), \\
I_{\mathrm{GD}}(x, y) & =\left\{\begin{array}{ll}
1, & \text { if } x \leq y \\
y, & \text { if } x>y
\end{array},\right. \\
I_{\mathrm{RC}}(x, y) & =1-x+x y, \\
I_{\mathrm{DN}}(x, y) & =\max (1-x, y), \\
I_{\mathrm{GG}}(x, y) & =\left\{\begin{array}{ll}
1, & \text { if } x \leq y \\
\frac{y}{x}, & \text { if } x>y
\end{array},\right. \\
I_{\mathrm{RS}}(x, y) & =\left\{\begin{array}{ll}
1, & \text { if } x \leq y \\
0, & \text { if } x>y
\end{array},\right. \\
I_{\mathrm{FD}}(x, y) & = \begin{cases}1, & \text { if } x \leq y \\
\max (1-x, y), & \text { if } x>y\end{cases} \\
I_{\mathrm{WB}}(x, y) & =\left\{\begin{array}{ll}
1, & \text { if } x \leq 1 \\
y, & \text { if } x=1
\end{array},\right. \\
I_{\mathrm{DP}}(x, y) & =\left\{\begin{array}{ll}
y, & \text { if } x=1 \\
1-x, & \text { if } y=0 \\
1, & \text { if } x<1, y>0
\end{array} .\right.
\end{aligned}
$$

Definition 9 ([5]). Let $N$ and $D$ be a fuzzy negation and disjunction, respectively. We say that the pair $(D, N)$ satisfies the law of excluded middle $(L E M)$ if both

$$
D(N(x), x)=1, \quad x \in[0,1],
$$

and

$$
D(x, N(x))=1, \quad x \in[0,1] .
$$

Theorem 1 ([5]). Let $N$ be a fuzzy negation and $D$ fuzzy disjunction without zero divisors. The pair $(D, N)$ fulfils (LEM1) (or (LEM2)) if and only if $N=N_{1}$.

A fuzzy negation can be generated from a fuzzy implication by means of a simple dependence as in the following theorem.

Theorem 2 ([1], p. 18). Let I be a fuzzy implication. The function

$N_{I}:[0,1] \rightarrow[0,1]$, where

$$
N_{I}(x)=I(x, 0), \quad x \in[0,1]
$$

is a fuzzy negation.

\section{Implications generated by fuzzy conjunction, disjunction and negation}

In this section we will use the law of classical propositional calculus indicating a dependance between a fuzzy implication and fuzzy conjunction, disjunction and negation

$$
(p \rightarrow q) \Leftrightarrow[\neg p \vee(p \wedge q)]
$$

to define some class of fuzzy implications. Such a way of defining fuzzy implication was introduced on the basis of quantum logic in the case when the conjunction is a triangular norm and the disjunction is a triangular conorm.

Definition 10. Let $C, D, N$ be a fuzzy conjunction, disjunction and negation, respectively. The function of the form

$$
I_{C, D, N}(x, y)=D(N(x), C(x, y)), \quad x, y \in[0,1]
$$

is called a $(C, D, N)$-operation.

Theorem 3. Let $I_{C, D, N}$ be a $(C, D, N)$-operation. Then the function $I_{C, D, N}$ is increasing with respect to the second variable and fulfils (1).

Proof. Monotonicity of the function $I_{C, D, N}$ with respect to the second variable is induced by monotonicity of the functions $C$ and $D$. Indeed, let $x, y_{1}, y_{2} \in[0,1], y_{1} \leqslant y_{2}$. We have $C\left(x, y_{1}\right) \leqslant C\left(x, y_{2}\right)$, and then $D\left(N(x), C\left(x, y_{1}\right)\right) \leqslant$ $D\left(N(x), C\left(x, y_{2}\right)\right)$, which proves that $I_{C, D, N}$ is increasing with respect to the second variable. Moreover

$$
\begin{aligned}
& I_{C, D, N}(1,1)=D(N(1), C(1,1))=D(0,1)=1, \\
& I_{C, D, N}(1,0)=D(N(1), C(1,0))=D(0,0)=0, \\
& I_{C, D, N}(0,0)=D(N(0), C(0,0))=D(1,0)=1 .
\end{aligned}
$$

By the above observed monotonicity we have $1 \geqslant$ $I_{C, D, N}(0,1) \geqslant I_{C, D, N}(0,0)=1$, which proves that $I_{C, D, N}(0,1)=1$. 
Example 5 (cf. [1], p. 91). Not any $(C, D, N)$ operation is a $(C, D, N)$-implication even for a triangular norm $C$ and triangular conorm $D$. Let us consider a function generated by the triple $\left(T_{M}, S_{M}, N_{\mathrm{S}}\right)$. For arbitrary $x, y \in[0,1]$ we have

$$
\begin{gathered}
I_{T_{M}, S_{M}, N_{\mathrm{S}}}(x, y)=S_{M}\left(N_{\mathrm{S}}(x), T_{M}(x, y)\right) \\
=\max (1-x, \min (x, y)) .
\end{gathered}
$$

Now let $x_{1}=0.5, x_{2}=0.6, y=0.9$. In this case

$$
\begin{gathered}
I_{T_{M}, S_{M}, N_{\mathrm{S}}}\left(x_{1}, y\right)=S_{M}\left(N_{\mathrm{S}}(0.5), T_{M}(0.5,0.9)\right) \\
=\max (0.5,0.5)=0.5, \\
I_{T_{M}, S_{M}, N_{\mathrm{S}}}\left(x_{2}, y\right)=S_{M}\left(N_{\mathrm{S}}(0.6), T_{M}(0.6,0.9)\right) \\
=\max (0.4,0.6)=0.6,
\end{gathered}
$$

which indicates that the function $I_{T_{M}, S_{M}, N_{\mathrm{S}}}$ is not decreasing with respect to the first variable, so it is not a fuzzy implication.

Definition 11. Let $I_{C, D, N}$ be a $(C, D, N)$ operation. If the function $I_{C, D, N}$ is decreasing with respect to the first variable, then it is called $(C, D, N)$-implication.

Theorem 4. Let $C, D$ be a fuzzy conjunction and disjunction, respectively and $N=N_{1}$. The function $I_{C, D, N}$ given by formula (3) is a $(C, D, N)$ implication.

Proof. It is enough to show that the function defined by (3) is decreasing with respect to the first argument. Let $x, y \in[0,1]$. Then

$$
\begin{gathered}
I_{C, D, N_{1}}(x, y)=D\left(N_{1}(x), C(x, y)\right) \\
= \begin{cases}D(0, C(x, y)), & \text { if } x=1 \\
D(1, C(x, y)), & \text { if } x<1\end{cases} \\
= \begin{cases}D(0, C(1, y)), & \text { if } x=1 \\
1, & \text { if } x<1\end{cases}
\end{gathered}
$$

So, regardless to the values of the function $I_{C, D, N_{1}}$ for $x=1$ the function is decreasing with respect to the first variable.

Let us observe that in the case when $C$ and $D$ have their left neutral elements 1 and 0 respectively, we have $I_{C, D, N_{1}}=I_{W B}$.

Example 6. The Table 1 presents examples of $(C, D, N)$-implications together with their suitable generators: $C$ - fuzzy conjunction, $D$ - fuzzy disjunction, $N$ - fuzzy negation. The symbol '-' means the function $I_{C, D, N}$ is not a fuzzy implication.

For example we present the way of generating the greatest fuzzy implication $I_{1}$. Let $N_{1}, C_{1}$ and $D_{1}$

\begin{tabular}{|c|c|c|c|}
\hline$C$ & $D$ & $N$ & $I_{C, D, N}$ \\
\hline$C_{0}$ & $D_{0}$ & $N_{0}$ & - \\
\hline$C_{1}$ & $D_{1}$ & $N_{1}$ & $I_{1}$ \\
\hline$T_{L K}$ & $S_{L K}$ & $N_{\mathrm{S}}$ & $I_{D N}$ \\
\hline$T_{P}$ & $S_{L K}$ & $N_{\mathrm{S}}$ & $I_{R C}$ \\
\hline$T_{P}$ & $S_{D}$ & $N_{\mathrm{S}}$ & $I_{D P}$ \\
\hline$T_{M}$ & $S_{L K}$ & $N_{\mathrm{S}}$ & $I_{L K}$ \\
\hline$T_{M}$ & $S_{D}$ & $N_{\mathrm{S}}$ & $I_{D P}$ \\
\hline$T_{M}$ & $S_{n M}$ & $N_{\mathrm{S}}$ & $I_{F D}$ \\
\hline
\end{tabular}

Table 1: $(C, D, N)$-implications.

be the greatest fuzzy negation, conjunction and disjunction, respectively. Then

$$
\begin{gathered}
I_{C_{1}, D_{1}, N_{1}}(x, y)=D_{1}\left(N_{1}(x), C_{1}(x, y)\right) \\
= \begin{cases}D_{1}\left(0, C_{1}(x, y)\right), & \text { if } x=1 \\
D_{1}\left(1, C_{1}(x, y)\right), & \text { if } x<1\end{cases} \\
= \begin{cases}D_{1}\left(0, C_{1}(1, y)\right), & \text { if } x=1 \\
1, & \text { if } x<1\end{cases} \\
= \begin{cases}0, & \text { if } x=1, y=0 \\
1 & \text { otherwise }\end{cases} \\
=I_{1}(x, y)
\end{gathered}
$$

for $x, y \in[0,1]$.

Let us now consider some properties of $(C, D, N)$ implications.

Example 7. Let us observe, that $(C, D, N)$ operators usually do not fulfil property (NP). Let $N, D$ be a fuzzy negation and disjunction, respectively, $C_{0}$ be the least fuzzy conjunction. Then for any $y \in(0,1)$ we have

$$
\begin{gathered}
I_{C_{0}, D, N}(1, y)=D\left(N(1), C_{0}(1, y)\right) \\
=D(0,0)=0 \neq y .
\end{gathered}
$$

Theorem 5. Let $N, D$ be a fuzzy negation and disjunction, respectively, $C$ be a fuzzy conjunction with the left neutral element 1 . The function $I_{C, D, N}$ fulfils property (NP) if and only if 0 is the left neutral element of a fuzzy disjunction D.

Proof. Let $y \in[0,1]$. Then we have

$$
I_{C, D, N}(1, y)=D(N(1), C(1, y))=D(0, y) .
$$

Thus, $I_{C, D, N}(1, y)=y$ if and only if $D(0, y)=y$.

Theorem 6. Let $N, C$ be a fuzzy negation and conjunction, respectively, $D$ be a fuzzy disjunction with the left neutral element 0 . The function $I_{C, D, N}$ fulfils property (NP) if and only if 1 is the left neutral element of a fuzzy conjunction $C$. 
Proof. Let $y \in[0,1]$. Then

$$
\begin{gathered}
I_{C, D, N}(1, y)=D(N(1), C(1, y))= \\
=D(0, C(1, y))=C(1, y) .
\end{gathered}
$$

Hence, we obtain $I_{C, D, N}(1, y)=y$ if and only if $C(1, y)=y$.

Theorem 7. Let $C$, $D$ be a fuzzy conjunction and disjunction, respectively, $N=N_{1}$. The function $I_{C, D, N}$ is a fuzzy implication satisfying property (IP).

Proof. From Theorem 4 it follows that $I_{C, D, N_{1}}$ is a fuzzy implication. Moreover, for an arbitrary $x \in$ $[0,1]$ we have

$$
\begin{gathered}
I_{C, D, N_{1}}(x, x)=D\left(N_{1}(x), C(x, x)\right) \\
= \begin{cases}D(0, C(1,1)), & \text { if } x=1 \\
D(1, C(x, x)), & \text { if } x<1\end{cases} \\
= \begin{cases}D(0,1), & \text { if } x=1 \\
1, & \text { if } x<1\end{cases} \\
=1 .
\end{gathered}
$$

The following theorem shows how to "regain" a fuzzy negation from $(C, D, N)$-implication.

Theorem 8. Let $C, D, N$ be a fuzzy conjunction, disjunction and negation, respectively, such that $I_{C, D, N}$ is decreasing with respect to the first argument. $N_{I_{C, D, N}}=N$ if and only if $D(x, 0)=x$ for $x \in N([0,1])$.

Proof. Let $y \in[0,1]$. By the use of formula (2) we obtain as follows

$$
\begin{gathered}
N_{I_{C, D, N}}(y)=I_{C, D, N}(y, 0) \\
=D(N(y), C(y, 0))=D(N(y), 0) .
\end{gathered}
$$

Thus, $N_{I_{C, D, N}}(y)=N(y)$ if and only if $D(N(y), 0) \stackrel{=}{=} N(y)$, that is if $D(x, 0)=x$ for $x \in N([0,1])$.

Theorem 9. Let $D, N$ be a fuzzy disjunction and negation, respectively, $C$ be a fuzzy conjunction with right neutral element 1 . If the function $I_{C, D, N}$ is a fuzzy implication then the pair $(D, N)$ fulfils the condition (LEM1).

Proof. Let $x \in[0,1]$. By Corollary 3 we have

$1=I_{C, D, N}(x, 1)=D(N(x), C(x, 1))=D(N(x), x)$.

Hence, $D(N(x), x)=1$.

From the above we obtain another result.

Theorem 10. Let $C, D, N$ be a fuzzy conjunction with the right neutral element 1, a fuzzy disjunction without zero divisors and a fuzzy negation, respectively. The function $I_{C, D, N}$ is a fuzzy implication if and only if $N=N_{1}$.
Proof. $(\Rightarrow)$ Let us assume that the function given by the formula (3) is a fuzzy implication. By Theorem 9 the pair $(D, N)$ fulfils the condition (LEM1). Thus $N=N_{1}$ on the basis of the Theorem 1 .

$(\Leftarrow)$ Now let $N=N_{1}$. Then by Theorem 4 the function $I_{C, D, N_{1}}$ is a fuzzy implication.

\section{Implications generated by two implications}

Now let us consider the generalization of the law in the classical propositional calculus:

$$
(p \Rightarrow q) \Leftrightarrow[p \Rightarrow(p \Rightarrow q)]
$$

Theorem 11 ([4], p. 75, cf. [9]). For any fuzzy implications $J, K$ the function of the form

$$
I_{J, K}(x, y)=J(x, K(x, y)), \quad x, y \in[0,1]
$$

is a fuzzy implication.

Proof. Let us observe first that

$$
\begin{aligned}
& I_{J, K}(1,1)=J(1, K(1,1))=J(1,1)=1, \\
& I_{J, K}(1,0)=J(1, K(1,0))=J(1,0)=0, \\
& I_{J, K}(0,1)=J(0, K(0,1))=J(0,1)=1, \\
& I_{J, K}(0,0)=J(0, K(0,0))=J(0,1)=1 .
\end{aligned}
$$

Moreover, the function $I_{J, K}$ is increasing with respect to the second variable as it is a composition of two increasing functions with respect to the second variable. Let us verify monotonicity with respect to the first variable. Let $x_{1}, x_{2}, y \in[0,1], x_{1} \leqslant x_{2}$. By monotonicity of the implication $K$ we obtain $K\left(x_{2}, y\right) \leqslant K\left(x_{1}, y\right)$, and then

$J\left(x_{2}, K\left(x_{2}, y\right)\right) \leqslant J\left(x_{2}, K\left(x_{1}, y\right)\right) \leqslant J\left(x_{1}, K\left(x_{1}, y\right)\right)$.

Thus, $I_{J, K}\left(x_{2}, y\right) \leqslant I_{J, K}\left(x_{1}, y\right)$, which ends the proof.

Definition 12. Let $J, K$ be fuzzy implications. The function $I_{J, K}$ given by (4) will be called $(J, K)$ implication.

Example 8. The above table shows examples of $(J, K)$-implications together with their generators.

\begin{tabular}{|c|c|c|}
\hline$J$ & $K$ & Implication $I_{J, K}$ \\
\hline$I_{\alpha}, \alpha \in[0,1]$ & $I_{\alpha}$ & $I_{\alpha}$ \\
\hline$I_{G D}$ & $I_{G D}$ & $I_{G D}$ \\
\hline$I_{R S}$ & $I_{G D}$ & $I_{R C}$ \\
\hline$I_{R S}$ & $I_{R S}$ & $I_{R S}$ \\
\hline
\end{tabular}

Table 2: $(J, K)$-implications.

Let us now examine some properties of $(J, K)$ implications which were presented in Definition 8 .

Theorem 12 ([4], p. 76, cf. [9]). If $J, K$ are fuzzy implications satisfying the property (NP), then $(J, K)$-implication fulfils the same property. 
Proof. By the property (NP) for the implications $J$ and $K$ we have

$$
I(1, y)=J(1, K(1, y))=J(1, y)=y
$$

for any $y \in[0,1]$.

Theorem 13. If $I$ is a fuzzy implication satisfying property (EP), then $(I, I)$-implication fulfils the same property.

Proof. Let $x, y, z \in[0,1]$. From the formula (4) and the property (EP) for the fuzzy implication $I$ we have

$$
\begin{aligned}
& I_{J, K}\left(x, I_{J, K}(y, z)\right)=I_{J, K}(x, I(y, I(y, z))) \\
& =I(x, I(x, I(y, I(y, z)))) \\
& =I(x, I(y, I(x, I(y, z)))) \\
& =I(y, I(x, I(y, I(x, z)))) \\
& =I(y, I(y, I(x, I(x, z)))) \\
& =I_{J, K}(y, I(x, I(x, z)))=I_{J, K}\left(y, I_{J, K}(x, z)\right),
\end{aligned}
$$

which means that our $(I, I)$-implication fulfills (EP).

Theorem 14 ([4], p. 75, cf. [9]). If $J, K$ are fuzzy implications, and $K$ fulfills (IP), then $(J, K)$ implications fulfills (IP).

Proof. Let $x \in[0,1]$. By formula (4) and by Corollary 3 we obtain

$$
I_{J, K}(x, x)=J(x, K(x, x))=J(x, 1)=1
$$

which imply that the implication $I_{J, K}$ fulfills (IP).

Example 9. Let us observe that the implications $I_{I_{G D}, I_{G D}}, I_{I_{R S}, I_{R S}}$ inherit property $(\mathrm{OP})$ of their generators. However a $(J, K)$-implication usually do not fulfil property (OP) even if its generators $J$ and $K$ do. Let us consider $\left(I_{G G}, I_{G G}\right)$-implications. We have

$$
\begin{gathered}
I_{I_{G G}, I_{G G}}(x, y)=I_{G G}\left(x, I_{G G}(x, y)\right) \\
= \begin{cases}1, & \text { if } x \leq I_{G G}(x, y) \\
\frac{I_{G G}(x, y)}{x}, & \text { if } x>I_{G G}(x, y)\end{cases} \\
= \begin{cases}1, & \text { if } y \geqslant x^{2} \\
\frac{y}{x^{2}}, & \text { if } y<x^{2}\end{cases}
\end{gathered}
$$

for any $x, y \in[0,1]$. Let $x=0.5, y=0.3$. Then $I_{I_{G G}, I_{G G}}(x, y)=I_{I_{G G}, I_{G G}}(0.5,0.3)=1$, but $y<x$. Hence, $\left(I_{G G}, I_{G G}\right)$-implication does not fulfil (OP), despite the fact that $I_{G G}$ fulfils this condition.

\section{Conclusion}

In this contribution two ways of generating of fuzzy implication from other fuzzy connectives are considered. The first method, according to the formula (3), involves three kinds of weak fuzzy connectives: fuzzy conjunction, disjunction and negation. The results show some dependencies between properties of generators and properties of generated operation. There are still a lot of possibilities to complete these findings. The second method (formula (4)) uses two fuzzy implications as generators. Preservation of some properties are considered. There are other properties of fuzzy implications which can be examined, e.g. the law of left or right contraposition with respect to some fuzzy negation.

\section{References}

[1] M. Baczyński, B. Jayaram, Fuzzy implications, Springer, Berlin, 2008.

[2] I. Batyrshin and O. Kaynak, Parametric classes of generalized conjunction and disjunction operations for fuzzy modelling, IEEE Transactions on Fuzzy Systems, 7:586-595, IEEE, 1999.

[3] J. Drewniak, A. Król, A survey of weak connectives and the preservation of their properties by aggregations, Fuzzy Sets and Systems, 161:202215, Elsevier, 2010.

[4] A. Król, Słabe spójniki logiczne (Weak logical connectives), Ph.D. Thesis, Oficyna Wydawnicza Politechniki Warszawskiej, Warszawa, 2011.

[5] A. Król, Fuzzy negation and other weak fuzzy connectives, Communications in Computer and Information Science 298:355-364, Springer-Verlag Berlin Heidelberg, 2012.

[6] M. Mas, M. Monserrat, J. Torrens, Two types of implications derived from uninorms, Fuzzy Sets and Systems 158:2612-2626, Elsevier, 2007.

[7] Y. Shi, B. Van Gasse, D. Ruan, E.E. Kerre, On the first place antitonicity in QL-implications, Fuzzy Sets and Systems 159:2988-3013, Elsevier, 2008.

[8] Y. Shi, D. Ruan, E.E. Kerre, On the characterizations of fuzzy implications satisfying $I(x, y)=$ $I(x, I(x, y))$, Information Sciences 177:29542970, Elsevier, 2007.

[9] N.R. Vemuri, B. Jayaram, Fuzzy implications: Novel generation process and the consequent algebras. Communications in Computer and Information Science 298:365-374, Springer-Verlag Berlin Heidelberg, 2012. 